\title{
The Link between Periodontal Inflammation and Cardiovascular Disease: The Relative Role of the Host Response
}

\author{
Muna S Elburki*, Alla F Elhashane and Ameerah A Alhasi \\ Department of Periodontics, Faculty of Dentistry, University of Benghazi, Benghazi, Libya \\ *Corresponding author: Muna S Elburki, Department of Periodontics, Faculty of Dentistry, University of Benghazi, \\ Benghazi, Libya
}

\section{ARTICLE INFO}

Received: 晀 December 21, 2020

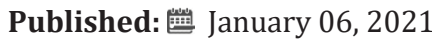

Citation: Muna S Elburki, Alla F Elhashane , Ameerah A Alhasi. The Link between Periodontal Inflammation and Cardiovascular Disease: The Relative Role of the Host Response. Biomed J Sci \& Tech Res 33(1)2021. BJSTR. MS.ID.005333.

Keywords: Chronic periodontitis; Cardiovascular disease; Systemic inflammation; Cholesterol; C-reactive protein; Matrix metalloproteinases

\section{ABSTRACT}

The role of periodontal disease in the etiology of cardiovascular disease has recently received considerable attention. Previous studies have shown conflicting results as to whether periodontitis is associated with increased risk of cardiovascular disease, however, numerous recent studies have demonstrated that patients with chronic destructive periodontitis show evidence of systemic inflammation which can link chronic periodontitis and cardiovascular disease. Moreover, periodontitis and cardiovascular disease, share the same risk factors such as smoking, male gender, aging and diabetes. The aim of the current study was to evaluate whether such an association exists and to explore the mechanistic link between local and systemic inflammation and the relative role of the host response. The current study adds to the strong evidence for an association between periodontitis and cardiovascular disease, however, does not prove causation. Further research is needed to clarify the strength of association between cardiovascular disease and periodontal disease.

Abbreviations: CVD: Cardiovascular Disease; MMPs: Matrix Metalloproteinases; P Gingivalis: Porphyromonas Gingivalis; HsCRP: High Sensitivity C-reactive Protein; LPS: Lipopolysaccharide; IL-6: Interleukin 6; LDL: Low-Density Lipoprotein; HDL: High-density Lipoprotein; BOP: Bleeding on Probing; PD: Probing Depth; CAL: Clinical Attachment level; SDD: Sub-antimicrobial Dose Doxycycline

\section{Introduction}

Periodontitis is chronic inflammatory diseases which is found worldwide and are among the most prevalent chronic infections in mankind. Severe forms of the disease can be found in $10-15 \%$ of the people and constitute a substantial inflammatory burden. People with advanced chronic periodontitis have been reported to have a significantly greater risk of developing cardiovascular disease (CVD), including atherosclerosis, myocardial infarction and stroke. Premature death in men is known to be significantly related with coronary heart disease. More and more research is pointing toward a possible link between periodontal disease and increased risk of CVD [1]. Periodontal disease is initiated by bacteria particularly anaerobic gram-negative microorganisms such as Porphyromonas gingivalis (P. gingivalis), Prevotella intermedia, Tannerella forsythia and others in the subgingival plaque or biofilm, or their microbial products notably lipopolysaccharide (LPS) or endotoxin (a constituent of the cell wall of these gram-negative bacteria), inducing inflammation in the adjacent gingival and periodontal tissues. However, it is now widely accepted that the collagen and connective tissue destruction of the gingiva and periodontal ligament, and the loss of alveolar bone, is largely mediated by the host response [2,3].

LPS and other substances enter the gingival tissues, initiate and propagate immuno-inflammation, leading to production of elevated levels of proinflammatory cytokines. These and other inflammatory mediators stimulate production of matrix metalloproteinases (MMPs) which degrade the connective tissues of the gingiva and periodontal ligament; these and prostaglandins facilitate alveolar bone resorption [4]. Several studies have demonstrated that patients with chronic destructive periodontitis show evidence of systemic inflammation as indicated by elevations of C-reactive 
protein (CRP), fibrinogen, interleukin 6 (IL-6), and other biomarkers in the circulation such as elevated cholesterol levels [5,6]. This systemic inflammation can link chronic periodontitis and CVD. In addition, periodontitis and certain systemic diseases, particularly cardiovascular disease, have the same risk factors such as tobacco smoking, male gender, race/ethnicity, stress, aging and diabetes [7].

The evidence is not clear yet, however, as stated by the American Academy of Periodontology, individuals with periodontal disease are nearly twice as likely to have coronary artery disease. In this regard one study found that the presence of common problems in the oral cavity, such as, gingivitis, caries, and missing teeth, were as good at predicting CVD disease as elevated cholesterol levels [8]. A link between periodontal disease and CVD may have important public health implications since both the periodontal disease and the CVD are highly prevalent in industrialized societies. Therefore, the link between periodontal disease and CVD is worth investigating because of its potential public health implications and because these studies could identify treatment strategies for periodontal disease that can interrupt this link. In addition, periodontal parameters including, bleeding on probing, Probing Depth (PD) and Clinical Attachment Level (CAL) may be a useful parameters of risk assessment for CVD in combination with other risk profile factors $[9,10]$.

\section{Materials and Methods}

This study included 120 subjects (50 male and 70 female) aged 31 to 95 years. The Patients were randomly selected from Outpatient Department (OPD) of the National Heart Center in Benghazi/ Libya. Ethical committee approval was obtained from Faculty of Dentistry, University of Benghazi, the nature and intention of the study was explained to the patients and an informed consent from each patient was obtained. A questionnaire contains information about each single patient included (Name, age, sex, history of heart disease and heart surgery, and some of important laboratory test like; Triglycerides, CRP levels) were included. The diagnosis of all cases based up on patient's medical history that was evaluated by qualified physician and some other laboratory investigations. Patients were excluded from the study if they had a known history of congenital heart disease.

A detailed case history which included information about the patient's overall medical status and oral status was recorded in a specially prepared form. Risk factors such as tobacco smoking, obesity, hypertension and diabetes mellitus was also recorded. Subjects were interviewed and underwent a complete examination of the oral cavity administered by a single periodontist with extensive experience. Teeth were counted and localized and a visual check for tooth mobility was performed. Assessment of periodontal status for all teeth present included measurement of probing depth (PD) in millimeters using a calibrated periodontal probe. Probing depth is the distance between the gingival margin and to the bottom of the gingival pocket measured at six sites of each tooth and deepest site was recorded. Gingival pockets $4 \mathrm{~mm}$ or deeper were pathogenic. Gingival inflammation was noted as bleeding on probing.

\section{Statistical Analysis}

The study used Statistical Package for Social Science (SPSS), version 18. Descriptive statistics including means, standard deviations, and percentages were used to summarize the results.

\section{Results}

A cross sectional study was undertaken with 120 participants to know the association between CVD and periodontal disease. Mean age of the participants was $58.65 \pm 10.21$ years, out of 120 subjects who participated in the study 70 were female and 50 were male. Out of the 120 participants, 44 of them have hypertension and diabetes mellitus, 39 have hypertension only and 11 have diabetes mellitus only. Smoking status was assessed as smoker and non-smoker. Out of the 120 participants, 33 of them were smokers and 87 were non-smokers. Obesity was also assessed as obese and non-obese. Out of the 120 participants, 39 of them were obese and 81were non-obese (Figure 1). Examination of the gingiva reveals bleeding on probing in $82 \%$ of the participants (Figure 2). Average tooth loss was $10.8 \pm 8.64$. Out of the 120 participants, 114 (95\%) have missing teeth and $3(2.5 \%)$ of them were edentulous (Figure 3). $98.3 \%$ of the subjects have probing pockets depth between $4 \mathrm{~mm}$ and $8 \mathrm{~mm}$ (Figure 4). Out of the 120 participants, $97.5 \%$ of them have some form of periodontal disease ranging from chronic gingivitis to chronic periodontitis (Table 1) and $90.6 \%$ of those cases have moderate to severe chronic periodontitis (Figure 5).

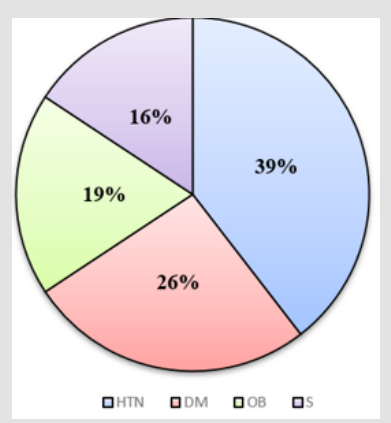

Figure 1: Risk Factors in Subjects with Diagnosed CVD: Hypertension (HTN), Diabetes Mellitus (DM), Obesity $(\mathrm{OB})$ and Smoking (S).

Table 1: Periodontal Disease in Subjects with Diagnosed CVD: The Number, Percentage, Mean Age and Standard Deviation.

\begin{tabular}{|c|c|c|c|c|}
\hline & Number & Percentage & $\begin{array}{c}\text { Mean } \\
\text { age }\end{array}$ & $\begin{array}{c}\text { Std } \\
\text { deviation }\end{array}$ \\
\hline Chronic Gingivitis & 2 & 1.70 & 31.5 & 0.5 \\
\hline Mild Periodontitis & 9 & 7.69 & 51.2 & 7.96 \\
\hline $\begin{array}{c}\text { Moderate } \\
\text { Periodontitis }\end{array}$ & 38 & 32.5 & 55.76 & 7.79 \\
\hline $\begin{array}{c}\text { Severe } \\
\text { Periodontitis }\end{array}$ & 68 & 58.11 & 61.3 & 9.75 \\
\hline
\end{tabular}




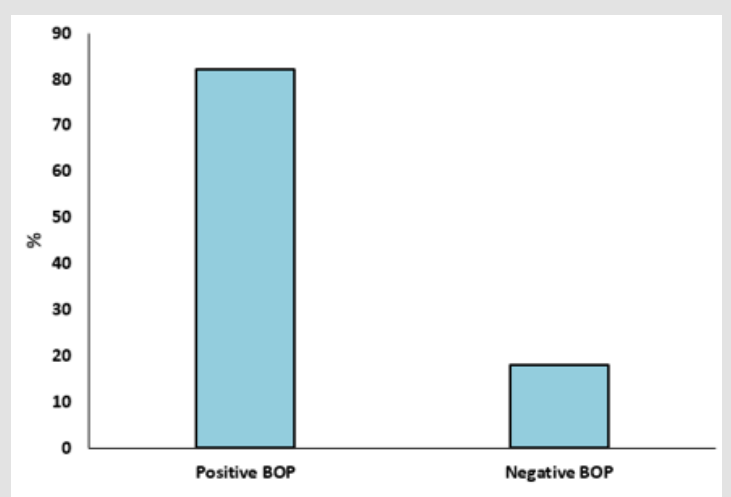

Figure 2: Bleeding on Probing (BOP) in Subjects with Diagnosed CVD.

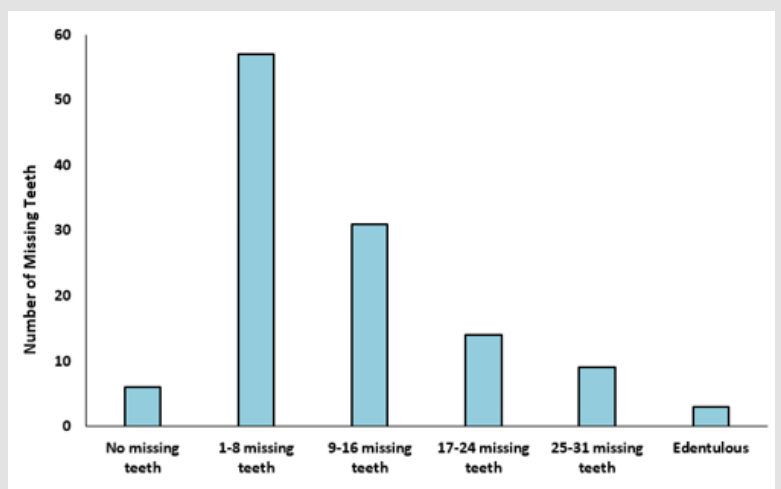

Figure 3: Number of Missing Teeth in in Subjects with Diagnosed CVD.

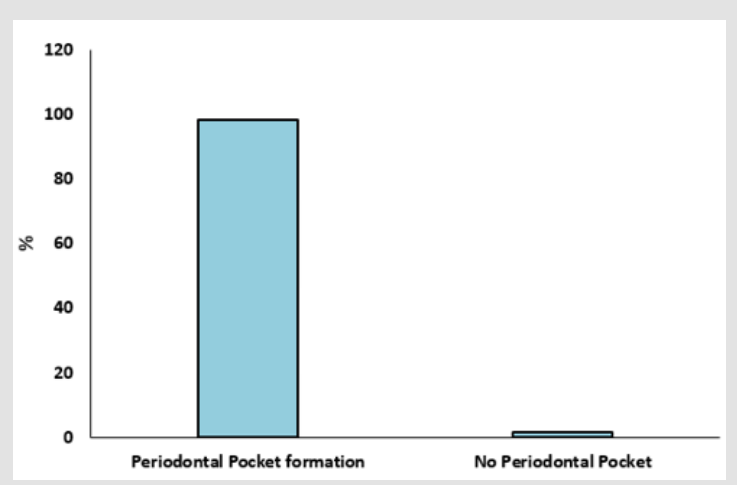

Figure 4: Periodontal Pocket Formation in Subjects with Diagnosed CVD.

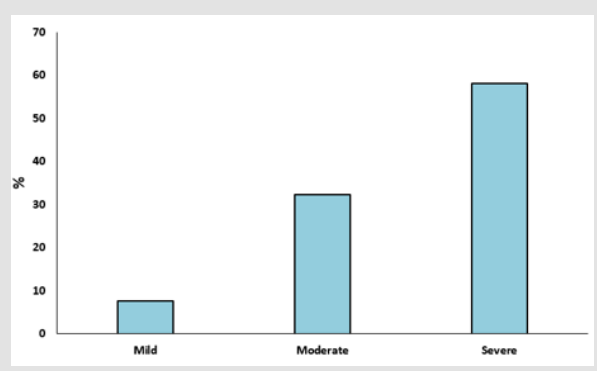

Figure 5: Severity of Chronic Periodontitis in Subjects with Diagnosed CVD.

\section{Discussion}

Periodontal disease is not only the most common of all chronic inflammatory diseases known to mankind, and the major cause of tooth loss in adults, it has also been linked to various medical diseases particularly CVD such as atherosclerosis. The following discussion initially addresses the epidemiological associations between CVD and periodontal disease. This section is followed by clinical trials linking periodontal inflammation to CVD and biological mechanisms for this link.

\section{Epidemiologic Studies}

Numerous recent studies have investigated the question of CVD as risk factors for periodontal disease [11-14]. Seymour et al. stated that despite 3000 years of history indicating an effect of oral disease on general health, it is only recently that the link between periodontal disease and CVD such as coronary heart disease and stroke, has been systematically studied. Regardless of these epidemiological links, the mechanisms for the different relationships have not yet been clarified, but are being investigated as described in this paper. Nevertheless, several hypotheses have been proposed, such as "common susceptibility, systemic inflammation with increased circulating cytokines and mediators, direct bacterial damage to the endothelium and cross-reactivity or molecular mimicry between bacterial antigens and self-antigens" [15].

Although not all populations or studies show statistically significant associations between periodontal diseases and CVD, several of the studies, which were summarized by "meta-analyses", indicate significant associations even after adjustment of the traditional risk factors such as smoking, race, gender, obesity and blood lipids. Whether these links are causally associated or because of fundamental genetic or behavioural risk factors that are shared by both conditions remains uncertain [16].

Pussinen et al. found that coronary artery disease was more prevalent in patients with antibodies to P. gingivalis compared to those who were antibody-negative after he analysed the link between coronary disease and antibody levels in response to $P$. gingivalis in severe chronic periodontitis $[17,18]$. Moreover, Noack et al. revealed a strong relationship between high serum levels of CRP, and the presence of numerous periodontal pathogenic bacteria including $P$. gingivalis in the subgingival biofilm [19]. The US National Health and Nutrition Study (NHANES) monitored patients for 17 years and concluded that in the presence of moderate to severe periodontitis the risk of coronary artery disease increased by $25 \%$ when compared to those patients with mild periodontal disease [20].

Recent studies also reported a positive correlation between the severity of periodontitis and atherosclerosis. The Atherosclerosis Risk in Communities (ARIC) Study provide the primary evidence that periodontitis may play a role in the pathogenesis of atheroma formation, in addition to cardiovascular events [21]. More recent 
studies of CVD association, using measurements that record clinical signs such as tooth loss and periodontal infectious and inflammatory measurements as exposures, generally indicate stronger links than just clinical parameters of disease. This point is vital to understand the link between the two diseases, as it is now largely believed that atherogenesis and plaque rupture, two important elements of cardiovascular pathogenesis that cause chronic disease burden and clinical events, are a result of systemic and vascular inflammatory processes [21,22].

\section{Clinical Trials Linking Periodontal Inflammation to Cardiovascular Disease}

In addition to the proposed role of periodontal pathogens in the progression of CVD, it is increasingly being recognized that the inflammatory host response mounted in both the periodontal and cardiovascular systems are likely responsible for the link between these two conditions. Recently it appears that chronic inflammation anywhere in the body such as rheumatoid arthritis, crohn's disease, chronic periodontitis, etc can increase the production of inflammatory mediators (e.g., prostanoids, nitric oxide, cytokines, and MMPs) at the local site, which can also be detected in the circulation. The pro-inflammatory cytokines in the plasma, such as IL-6, can stimulate the liver to produce acute phase proteins (such as CRP, and other inflammatory mediators, fibrinogen, haptoglobin, etc.) which are the systemic markers of inflammation which can link chronic periodontitis and CVD. Furthermore, to these inflammatory biomarkers, MMPs, with increased levels and activity of MMP-2, -8 and -9 , are also associated with both chronic periodontitis and CVD $[23,24]$.

Tuomainen et al. reported that there is a link between high levels of MMP-8 (neutrophil-type collagenase) in serum and cardiac events, and in addition to MMP8, MMP-9 as well is also elevated in gingival crevicular fluid (GCF) in periodontal lesions in patients with Chronic Periodontitis [25]. Moreover, Blankenberg et al. pointed out MMP-9, which is elevated in the periodontal pocket (gingival crevicular fluid) and gingiva in periodontitis [26], has been positively correlated with the incidence of myocardial infarction even fatal attacks when measured in plasma in long term clinical trials involving over 1.000 patients with CVD [27]. A high serum level of high-sensitivity CRP (HsCRP) is a biomarker of systemic inflammation and significantly increases the risk for CVD in combination with other risk profile factors [28,29].

Recent studies have demonstrated that severe chronic periodontitis may also cause elevated serum levels of CRP $[5,19,30]$. In addition, some studies (but not all) have shown that periodontal treatment can reduce the serum levels of these biomarkers of systemic inflammation such as CRP [28,29,31]. Elevated plasma levels of acute-phase proteins such as CRP, fibrinogen, serum amyloid A, and IL-6 have been reported in periodontitis in human subjects. These findings propose that recruitment of the systemic inflammatory response machinery may complement localized diseases of the periodontium [32]. Although several studies have shown association between periodontal disease and CVD, there have been only a few studies to address the potential benefit of periodontal treatment on the markers of cardiovascular risk or cardiovascular outcomes. Recent studies have suggested that periodontal therapy can reduce levels of serum HsCRP, lower IL6 , and improve endothelial function as measured by flow-mediated dilation [33]. Tuter et al. reported that combination therapy (Scaling and root planning [SRP]+ Sub-antimicrobial dose Doxycycline i.e.., SDD) produced statistically significant benefits in both local periodontal disease (pocket depth, and gingival index) and systemic biomarkers (high-density lipoprotein [HDL] cholesterol and APO-A, lipoprotein core of HDL) compared with (SRP+ placebo) in patients with both chronic periodontitis and coronary artery disease [34].

Recently Salminen et al. in a study involving 45 postmenopausal women who exhibited both periodontal disease and systemic bone loss, demonstrated that the serum from those women on a 2 year regimen of SDD showed a significant increase in cholesterol efflux from macrophages in culture compared to those postmenopausal women treated with placebo. These finding identified one mechanism by which SDD therapy, in subjects vulnerable to CVD, can reduce the formation of cholesterol-rich atheroscleromatous plaques [35]. Brown et al. used the same FDA-approved treatment for chronic periodontitis, i.e.., SDD as an MMP-inhibitor, in patients with severe CVD, acute coronary syndromes (i.e.., history of myocardial infarction, angina and 1,2 or 3 vessel atherosclerosis). The subjects were administered, in a double-blind placebocontrolled study, SDD bid (or look-like placebo) for 6 months. He found that SDD treatment provided a significant reduction in MMP-9, HsCRP and IL-6 in the plasma of these patients compared to the placebo-treated subjects, indicating decreased systemic inflammation and decreased risk for future cardiac event without treating periopathogens [36]. It should be noted that Brown et al. did not examine the periodontal status of those severely diseased CVD patients; however, it should also be appreciated that patients with this severe form of CVD, acute coronary syndromes, have recently been found to universally exhibit severe periodontal disease. Again, these observations support the well-known link between periodontal disease, systemic inflammation, and severe medical diseases notably CVD. Both Gotsman et al. and Gu et al. reported a near-100\% incidence of periodontitis in patients with acute coronary syndromes, and that their blood levels of systemic inflammation (HsCRP) appeared to be elevated despite traditional statin therapy [24,37].

\section{Biological Mechanisms}

Many older medical studies have suggested a role in CVD for infection with a variety of organisms, such as Chlamydia pneumoniae, cytomegalovirus, and others. As the exact role of these infections in the development and progression of atherosclerosis is not completely understood, it is reasonable to suggest that 
chronic periodontal infections could also take part in this multiple hit model, accelerating mechanisms involved in atherogenesis. However, recent multi-institutional studies both in the USA and Europe have found that long-term antimicrobial therapy had no effect on CVD [38-41], so in cardiology (if not in periodontics), this hypothesis has been overtaken by the "systemic inflammation" hypothesis.

In periodontitis, damage of the attachment apparatus of the teeth is started by bacterial colonization of root surfaces and release of bacterial substances onto the epithelial lining of the gingival crevice. Once bacteria colonize the pocket, their byproducts provoke periods of exacerbation and remission of inflammation, resulting in increased production of proinflammatory cytokines and matrix metalloproteinases. A consequence of this heavy load of cytokines and tissue-destructive mediators is the development of hyperpermeability and loss of epithelial and basement membrane integrity, which creates a chance for assaulting bacteria, and their byproducts, to enter the systemic circulation. Ultimate support for the idea that oral infection may involve the systemic host response is apparent from the observation that specific antibody responses to oral bacteria appear on infection. Moreover, such antibodies could, possibly, generate endothelial cytotoxic effects, as stated in the case of antibodies to Escherichia coli and C pneumoniae. Significantly, bacteria and release of LPS into the circulation may act as systemic triggers, stimulating a range of cytokines and tissue-destructive mediators in circulating inflammatory effector cells [32].

The detection of elevated serum levels of LPS or antibodies to P. gingivalis is associated with a higher risk of atherosclerosis development in humans. Therefore, although several factors contribute to atherosclerosis development, inflammation resulting from LPS exposure may aggravate the disease [42]. Li etal. reported that long-term systemic challenge with $P$. gingivalis, can accelerate atherogenic plaque development in a heterozygous Apolipoprotein E-deficient murine model. He found that the atherosclerotic lesions of the proximal aortas and aortic trees were more progressive in animals challenged with $P$. gingivalis than in vehicle control animals [43], however, this effect of $P$. gingivalis may be mediated by systemic inflammation. Moreover, Jain et al. demonstrated that animals with experimentally induced periodontitis had more extensive accumulations of lipids inside the aorta than in control animals and there was a strong correlation between the extent of lipid deposition and the severity of periodontitis.

He concluded that infections at remote locations can modulate atherosclerotic events distantly [44]. In addition, Hayashi et al demonstrated that by using in vivo MRI analysis together with ex vivo immunohistochemistry, P. gingivalis exposure results in an increase of atherosclerotic plaque accumulation in the arterys of ApoE deficient mice that is associated with the accumulation of lipids and macrophages. Furthermore, he also found increases in mean plaque area, lipids, and macrophage accumulation were prevented by immunization via a heat-killed preparation of $P$. gingivalis prior to challenge with live bacteria [45]. Gitlin and Loftin reported that $P$. gingivalis significantly increased atherosclerosis development in ApoE deficient mice when LPS was administered by chronic infusion for 28 days [3]. Although periodontal research has largely focused on the role of periopathogens, such as P. gingivalis, in the link between chronic periodontitis and CVD, as described earlier, cardiology research has largely moved away from this concept, and focused on systemic inflammation and lipid (e.g., cholesterol, triglyceride) abnormalities and elevated MMPs, as the driving force for CVD and as a target for therapeutic strategies (e.g., cholesterollowering statin, anti-inflammatory agents, resolving, etc).

However, in recent years, contribution of the host response, rather than periodontopathogens, may be the primary contributor to the link between periodontal disease and CVD. Cytokines produced locally, in inflamed periodontal tissues, such as IL- 6 can be carried by the circulation to the liver where they induce the expression of acute phase proteins such as C-reactive protein, fibrinogen and haptoglobin resulting in the systemic inflammatory response [46]. CRP can form a complex with oxidized low-density lipoprotein (LDL) cholesterol, which, when phagocytosed by macrophages invading atheroscleromatous plaques can differentiate into foam cells $[47,48]$. The production of matrix metalloproteinases, for instance MMP-8 and MMP-9 by these inflammatory cells, can destroy the thin collagen "cap" that covers cholesterol- rich plaques lining the coronary arteries leading to plaque rupture, leading to thrombosis and acute myocardial infarction [48-50].

In summary, the results of the present study demonstrated a significant association between periodontal disease and CVD and indicated a near-100\% incidence of periodontal disease in patients with CVD and have shown that almost all CVD patients exhibited moderate-severe chronic periodontitis. Although there was a positive correlation between periodontal disease and an increased risk for CVD, we cannot exclude, however, that general risk factors such as tobacco smoking, male gender, race/ethnicity, stress, aging and diabetes has an effect on the severity of periodontal disease and also considered risk factors for CVD. Periodontal disease was significantly correlated with CVD, therefore, periodontal parameters including, bleeding on probing (BOP), Probing Depth (PD) and Clinical Attachment Level (CAL) may be a useful parameters of risk assessment for CVD. A high serum level of high-sensitivity CRP (HsCRP) is a biomarker of systemic inflammation and significantly increases the risk for CVD [51,52] and might be useful as a method of risk assessment for CVD. However, CVD cannot be diagnosed with certainty by using HsCRP, therefore, periodontal parameters and HsCRP could be used in combination with other risk factors.

Our approach has been to consider the situation when the dentist detects severe periodontitis (PPD $\geq 7 \mathrm{~mm}$ ) and must decide whether this patient needs referral for specialist investigation. From the literature we know that Severe periodontitis are strongly 
correlated with CVD, Therefore, they could be used in combination with other risk factors such as obesity, tobacco smoking, male gender, race/ethnicity, stress, advancing age, hypertension and in particular, diabetes and at least if there is one positive dental risk factor and one positive general risk factor the patient should be referred for consultation. Additional research is necessary to elucidate the strength of association between CVD and periodontal disease. A longitudinal assessment of systemic inflammatory markers and changes in periodontal parameters would be the preferred method for quantifying the association between CVD and periodontal disease. Nevertheless, the present study does fit well with in the findings of the existing literature.

\section{Acknowledgments}

The authors would like to thank Dr. Marwan Alwerfaly and Dr. Mohammed Ahbara, for their assistance. The kind assistance of the clinical staff of the National Heart Center in Benghazi/Libya, is gratefully acknowledged.

\section{References}

1. Katz J, Chaushu G, Sharabi Y (2001) On the association between hypercholesterolemia, cardiovascular disease and severe periodontal disease. J Clin Periodontol 28(9): 865-868.

2. Ryan M (2002) Host modulation: conceptualization to clinical trials and integration into clinical practice. J Calif Dent Assoc 30(4): 285-288, 290-295.

3. Gitlin JM, Loftin CD (2009) Cyclooxygenase-2 inhibition increases lipopolysaccharide-induced atherosclerosis in mice. Cardiovasc Res 81(2): 400-407.

4. Page R (1998) The Pathobiology of Periodontal Diseases May Affect Systemic Diseases: Inversion of a Paradigm. Ann Periodontol 3(1): 108 120.

5. Craig R, Yip J, So M, Boylan R, Socransky S (2003) Relationship of destructive periodontal disease to the acute-phase response. J Periodontol 74(7): 1007-1016

6. Slade G, Ghezzi E, Heiss G, Beck J, Riche E, et al. (2003) Relationship between periodontal disease and C-reactive protein among adults in the Atherosclerosis Risk in Communities study. Arch Intern Med 163(10): 1172-1179

7. Demmer RT, Desvarieux M (2006) Periodontal infections and cardiovascular disease The heart of the matter. J Am Dent Assoc 137(suppl 2): 14S-20S.

8. Meurman JH, Sanz M, Janket SJ (2004) Oral health, atherosclerosis, and cardiovascular disease. Crit Rev Oral Biol Med 15(6): 403-413.

9. D Aiuto F, Parkar M, Andreou G, J Suvan, Peter M, et al. (2004) Periodontitis and Systemic Inflammation: Control of the Local Infection is Associated with a Reduction in Serum Inflammatory Markers. J Dent Res 83(2): 156-160.

10. Ridker P, Danielson E, Fonseca F, Libby P (2008) Rosuvastatin to Prevent Vascular Events in Men and Women with Elevated C-Reactive Protein. N Engl J Med 359(21): 2195-2207.

11. Paquette DW, Bell KP, Phillips C, Offenbacher S, Wilder RS (2015) Dentists' knowledge and opinions of oral-systemic disease relationships: relevance to patient care and education. J Dent Educ 79(6): 626-635.

12. Kim J, Amar S (2006) Periodontal disease and systemic conditions: a bidirectional relationship. Odontology 94(1): 10-21.

13. Garcia RI, Henshaw MM, Krall EA (2001) Relationship between periodontal disease and systemic health. Periodontol 2000 25(1): 21-36.
14. Genco RJ, Loe H (1993) The role of systemic conditions and disorders in periodontal disease. Periodontol 2000 2: 98-116.

15. Seymour GJ, Ford PJ, Cullinan MP, Leishman S, Yamazaki K (2007) Relationship between periodontal infections and systemic disease. Clin Microbiol Infect 13(s4):3-10.

16. Bahekar AA, Singh S, Saha S, Molnar J, Arora R (2007) The prevalence and incidence of coronary heart disease is significantly increased in periodontitis: A meta-analysis. Am Heart J 154(5): 830-837.

17. Pussinen PJ, Alfthan G, Rissanen H, Reunanen A, Asikainen S, et al. (2004) Antibodies to Periodontal Pathogens and Stroke Risk. Stroke 35(9): 2020-2023.

18. Pussinen PJ, Jousilahti P, Alfthan G, Timo P, Sirkka A, et al. (2003) Antibodies to Periodontal Pathogens Are Associated With Coronary Heart Disease. Arterioscler Thromb Vasc Biol 23(7): 1250-1254.

19. Noack B, Genco RJ, Trevisan M, Grossi S, Zambon J, et al. (2001) Periodontal infections contribute to elevated systemic C-reactive protein level. J Periodontol 72(9): 1221-1227.

20. DeStefano F, Anda RF, Kahn HS, Williamson DF, Russell CM (1993) Dental disease and risk of coronary heart disease and mortality. BMJ 306(6879): 688.

21. Beck JD, Elter JR, Heiss G, Couper D, Mauriello SM, et al. (2001) Relationship of Periodontal Disease to Carotid Artery Intima-Media Wall Thickness: The Atherosclerosis Risk in Communities (ARIC) Study. Arterioscler Thromb Vasc Biol 21(11): 1816-1822.

22. Desvarieux M, Demmer RT, Rundek T, BB Albala, David R, et al. (2003) Relationship Between Periodontal Disease, Tooth Loss, and Carotid Artery Plaque: The Oral Infections and Vascular Disease Epidemiology Study (INVEST). Stroke 34(9): 2120-2125.

23. Elburki MS, Moore DD, Terezakis NG, Y Zhang, HM Lee, et al. (2017) A novel chemically modified curcumin reduces inflammation-mediated connective tissue breakdown in a rat model of diabetes: periodontal and systemic effects. J Periodontal Res 52(2): 186-200.

24. Gu Y, Lee HM, Sorsa T, Aino S, Maria E, et al. (2011) Non-antibacterial tetracyclines modulate mediators of periodontitis and atherosclerotic cardiovascular disease: A mechanistic link between local and systemic inflammation. Pharmacol Res 64(6): 573-579.

25. Tuomainen AM, Nyyssonen K, Laukkanen JA, Tania T, Tomi P, et al. (2007) Serum Matrix Metalloproteinase-8 Concentrations Are Associated with Cardiovascular Outcome in Men. Arterioscler Thromb Vasc Biol 27(12): 2722-2728.

26. Lee HM, Ciancio S, Tuter G, Ryan M, Komaroff E, et al. (2004) Subantimicrobial dose doxycycline efficacy as a matrix metalloproteinase inhibitor in chronic periodontitis patients is enhanced when combined with a non-steroidal anti-inflammatory drug. J Periodontol 75(3): 453463.

27. Blankenberg S (2003) Plasma Concentrations and Genetic Variation of Matrix Metalloproteinase 9 and Prognosis of Patients With Cardiovascular Disease. Circulation 107(12): 1579-1585.

28. D Aiuto F, Parkar M, Andreou G, J Suvan, Peter M, et al. (2004) Periodontitis and Systemic Inflammation: Control of the Local Infection is Associated with a Reduction in Serum Inflammatory Markers. J Dent Res 83(2): 156-160.

29. Ridker P, Danielson E, Fonseca F, Libby P (2008) Rosuvastatin to Prevent Vascular Events in Men and Women with Elevated C-Reactive Protein. N Engl J Med 359(21): 2195-2207.

30. D Aiuto F, Ready D, Tonetti MS (2004) Periodontal disease and C-reactive protein-associated cardiovascular risk. J Periodontal Res 39(4): 236241.

31. Redker P, Rifai N, Cook N, Bradwin G, Buring J (2005) Non-HDL Cholesterol, Apolipoproteins A-I and B100, Standard Lipid Measures, Lipid Ratios, and CRP as Risk Factors for Cardiovascular Disease in Women. JAMA 294(3): 326-333. 
32. Lalla E, Lamster I, Hofmann M, Loredana B, AP Jerud, et al. (2003) Oral Infection With a Periodontal Pathogen Accelerates Early Atherosclerosis in Apolipoprotein E-Null Mice. Arterioscler Thromb Vasc Biol 23(8): 1405-1411.

33. Offenbacher S, Beck JD, Moss K (2009) Results From the Periodontitis and Vascular Events (PAVE) Study: A Pilot Multicentered, Randomized, Controlled Trial to Study Effects of Periodontal Therapy in a Secondary Prevention Model of Cardiovascular Disease. J Periodontol 80(2): 190201.

34. Tüter G, Kurtiș B, Serdar M (2007) Effects of scaling and root planing and sub-antimicrobial dose doxycycline on oral and systemic biomarkers of disease in patients with both chronic periodontitis and coronary artery disease. J Clin Periodontol 34(8): 673-681.

35. Salminen A, Pussinen PJ, Payne JB, Julie AS, Matti J, et al. (2013) Subantimicrobial-dose doxycycline treatment increases serum cholesterol efflux capacity from macrophages. Inflamm Res 62(7): 711720.

36. Brown DL, Desai K, Vakili B, Nouneh C, Lee HM, et al. (2004) Clinical and Biochemical Results of the Metalloproteinase Inhibition with Subantimicrobial Doses of Doxycycline to Prevent Acute Coronary Syndromes (MIDAS) Pilot Trial. Arterioscler Thromb Vasc Biol 24(4): 733-738.

37. Gotsman I, Lotan C, Lapidus L (2007) Periodontal destruction is associated with coronary artery disease and periodontal infection with acute coronary syndrome. J Periodontol 78(5): 849-858.

38. Cannon CP, Braunwald E, McCabe CH, Thomas G, Brent M, et al. (2005) Antibiotic treatment of Chlamydia pneumoniae after acute coronary syndrome. N Engl J Med 352(16): 1646-1654.

39. Grayston JT, Kronmal RA, Jackson LA, Alfred F, Joseph B, et al. (2005) Azithromycin for the secondary prevention of coronary events. N Engl J Med 352(16): 1637-1645.

40. O Connor C, Dunne M, Pfeffer M (2003) Azithromycin for the Secondary Prevention of Coronary Heart Disease Events the WIZARD Study: A Randomized Controlled Trial. JAMA 290(11): 1459-1466.

41. Anderson JL, Muhlestein JB, Carlquist J, Ann A, Sanjeev T, et al. (1999) Randomized Secondary Prevention Trial of Azithromycin in Patients
With Coronary Artery Disease and Serological Evidence for Chlamydia pneumoniae Infection: The Azithromycin in Coronary Artery Disease: Elimination of Myocardial Infection with Chlamydia (ACADEMIC) Study. Circulation 99(12): 1540-1547.

42. Pussinen PJ, Tuomisto K, Jousilahti P, Havulinna AS, Sundvall J, et al. (2007) Endotoxemia, Immune Response to Periodontal Pathogens, and Systemic Inflammation Associate With Incident Cardiovascular Disease Events. Arterioscler Thromb Vasc Biol 27(6): 1433-1439.

43. Li L, Messas E, Batista E (2002) Porphyromonas gingivalis Infection Accelerates the Progression of Atherosclerosis in a Heterozygous Apolipoprotein E-Deficient Murine Model. Circulation 105(7): 861-867.

44. Jain A, Batista EL, Serhan C, Stahl GL, Van Dyke TE (2003) Role for Periodontitis in the Progression of Lipid Deposition in an Animal Model. Infect Immun 71(10): 6012-6018.

45. Hayashi C, Viereck J, Hua N (2011) Porphyromonas gingivalis accelerates inflammatory atherosclerosis in the innominate artery of ApoE deficient mice. Atherosclerosis 215(1): 52-59.

46. Mattilla K, Pussinen P, Paju S (2005) Dental infections and cardiovascular diseases: a review. J Periodontol 76(115): 2085-2088.

47. Østerud B, Bjørklid E (2003) Role of monocytes in atherogenesis. Physiol Rev 83(4): 1069-1112.

48. Elburki MS (2015) Periodontal Disease and Potential Association with Systemic Diseases and Conditions. Applied Clinical Research, Clinical Trials \& Regulatory Affairs 2(2): 73-79.

49. Libby $P$ (1995) Molecular bases of the acute coronary syndromes. Circulation 91(11): 2844-2850.

50. Lee R, Libby P (1997) The unstable atheroma. Arterioscler Thromb Vasc Biol 17(10): 1859-1867.

51. Stankovic B, Minic I (2019) The Role of Periodontal Disease in Etiology of Myocardial Infarction. Archives of Medicine 11(14): 1-4.

52. Muhsin J, Al-sayyid M (2019) The possible Connection of periodontal diseases (PD) with cardiovascular disease (CVD) and prostatitis in sample of Iraqi patients. Muthanna Medical Journal 6(1): 1-12.
ISSN: 2574-1241

DOI: 10.26717/BJSTR.2021.33.005333

Muna S Elburki. Biomed J Sci \& Tech Res

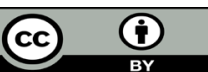

This work is licensed under Creative Commons Attribution 4.0 License

Submission Link: https://biomedres.us/submit-manuscript.php

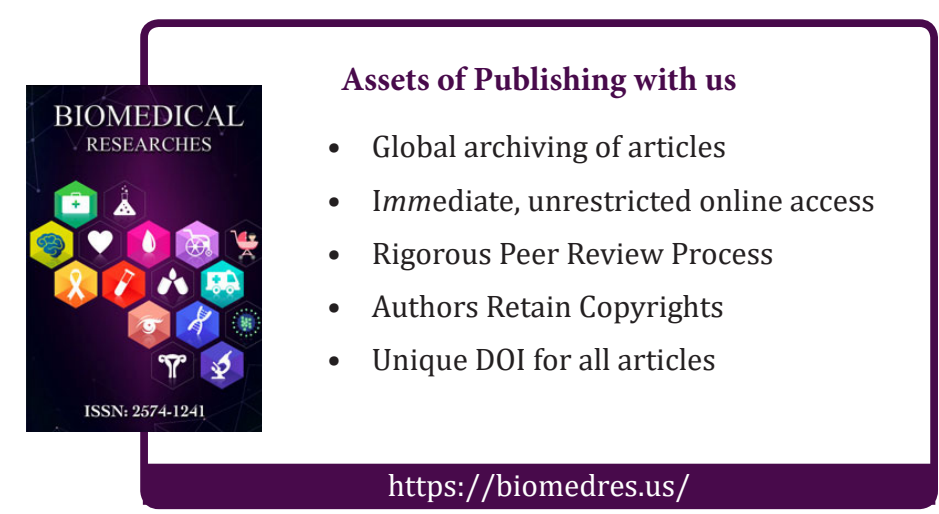

